\title{
Rapporter des souvenirs importuns : les voyages et les bactéries pharmacorésistantes
}

\author{
BJ Langford ${ }^{1,2}, \mathrm{KL} \mathrm{Schwartz}^{1,2,3 *}$
}

\section{Résumé}

La résistance aux antimicrobiens constitue une importante menace à la santé publique, tant à l'échelle internationale qu'au Canada. La grande variabilité de la résistance aux antimicrobiens que l'on observe d'une région à l'autre et l'augmentation actuelle des voyages internationaux entraînent un risque important d'acquisition et de transmission d'organismes pharmacorésistants. Les voyages de pays à revenu élevé vers des pays à revenu faible et moyen, particulièrement sur le sous-continent indien, présentent les risques les plus élevés d'acquisition d'entérobactéries pharmacorésistantes. Les facteurs de risque de contraction d'organismes pharmacorésistants lors d'un voyage comprennent les soins médicaux reçus à l'étranger, la diarrhée du voyageur et l'utilisation d'antibiotiques. Les professionnels de la santé peuvent jouer un rôle important dans la prévention des dangers pour la santé des voyageurs, en informant les patients au sujet du risque de contracter des organismes pharmacorésistants, ainsi qu'en prescrivant des antibiotiques lorsqui'ils sont indiqués contre la diarrhée du voyageur et un traitement empirique personnalisé pour les patients présentant une infection après un voyage.

\author{
Affiliations \\ ${ }^{1}$ Santé publique Ontario, Toronto \\ (Ontario) \\ ${ }^{2}$ Centre de santé St-Joseph, \\ Toronto (Ontario) \\ ${ }^{3}$ École de santé publique \\ Dalla Lana, Toronto (Ontario)
}

${ }^{\star}$ Correspondance :

kevin.schwartz@oahpp.ca

Citation proposée : Langford BJ, Schwartz KL. Rapporter des souvenirs importuns : les voyages et les bactéries pharmacorésistantes. Relevé des maladies transmissibles au Canada 2018;44(11):315-21. https://doi. org/10.14745/ccdr.v44i11a02f

Mots clés : résistance aux antimicrobiens, voyages, gestion de l'utilisation des antimicrobiens, prévention et contrôle des infections, tourisme médical

\section{Introduction}

La résistance aux antimicrobiens (RAM) est un problème croissant à l'échelle mondiale. Elle est considérée comme l'une des plus importantes menaces pour la santé publique de notre époque. En l'absence d'une intervention efficace, on estime que les décès attribuables aux infections pharmacorésistantes passeront de 700000 à 10 millions par année en 2050, dépassant le cancer comme la principale cause de décès (1). En outre, la prévalence des organismes pharmacorésistants varie grandement à travers le monde. Par exemple, la résistance d'Escherichia coli aux céphalosporines de troisième génération est beaucoup plus fréquente en Inde, à $78 \%$, qu'au Canada, à $9 \%(2)$.

Dans notre société mondialisée, la RAM n'a pas de frontières. En 2017, le nombre de passagers aériens a dépassé pour la première fois les quatre milliards, et ce nombre devrait doubler d'ici à 2036 (3).

Les infections par des entérobactéries pharmacorésistantes constituent l'un des grands défis de la RAM associée aux voyages. Les entérobactéries forment une grande famille de bacilles à gram négatif pouvant causer une grande variété d'infections, notamment celles qui touchent les voies urinaires, les voies respiratoires et le tractus gastro-intestinal. Cette famille compte parmi ses membres des organismes tels qu'E. coli, les espèces de Klebsiella, les espèces d'Enterobacter et les espèces de Salmonelle. Chez les entérobactéries, I'un des principaux mécanismes de résistance aux antibiotiques est la production de bêta-lactamases et de carbapénémases, des enzymes qui hydrolysent les antibiotiques de type bêta-lactamines, les rendant ainsi inefficaces. Les gènes de ces enzymes sont souvent encodés par des plasmides, qui peuvent passer d'une bactérie à l'autre. Les principaux groupes d'entérobactéries résistantes sont les entérobactéries productrices de bêta-lactamases à spectre étendu (EP-BLSE) et les entérobactéries résistantes aux carbapénèmes $(E R C)$. Le gène $m c r-1$ de résistance à la colistine à médiation plasmidique est de plus en plus important. La capacité des bactéries à échanger entre elles des gènes résistants aux antimicrobiens au moyen de plasmides pose de grands défis en matière de lutte contre les infections. Une augmentation de 
la résistance des entérobactéries se traduit par une hausse de la mortalité associée à des infections, par des hospitalisations prolongées et par une hausse des coûts imposés au système de soins de santé $(4,5)$.

L'objectif du présent article est de décrire le risque clinique des organismes pharmacorésistants associés aux voyages, en mettant l'accent sur les entérobactéries. Aux fins du présent article, les voyages sont définis comme tout déplacement de personnes vers des pays à revenu faible ou moyen d'Asie, d'Afrique ou des Amériques et leur retour à des pays à revenu élevé, comme l'Australie, le Canada, la Nouvelle-Zélande, les États-Unis (É-U) et les pays européens.

\section{Quel est le risque de rapporter des bactéries pharmacorésistantes au retour d'un voyage?}

Les études sur le risque pour les voyageurs de contracter des EP-BLSE ou des ERC donnent à réfléchir et présentent des considérations cliniques importantes pour la prise en charge des patients au Canada.

\section{Entérobactéries productrices de bêta- lactamases à spectre étendu}

L'une des plus importantes études d'évaluation du risque de contraction d'un organisme pharmacorésistant au cours d'un voyage a porté sur l'importation d'EP-BLSE dans les Pays-Bas. Dans le cadre d'une étude de cohorte longitudinale menée auprès de 2001 voyageurs, les auteurs ont déterminé la probabilité d'une colonisation par des EP-BLSE avant et après la période du voyage. Parmi les personnes qui ne présentaient pas d'EP-BLSE avant leur voyage, $34 \%$ ont contracté une EP-BLSE alors qu'elles étaient à l'étranger (6). On a observé une variabilité marquée du risque de colonisation par des EP-BLSE selon la région visitée. Les voyages en Asie du Sud présentaient le risque le plus élevé, avec une incidence de colonisation de $75 \%$; suivis par les voyages en Asie centrale et de l'Est (49\%), en Asie de l'Ouest (43\%), en Asie du Sud-Est (37\%), dans les Caraïbes et en Amérique centrale ( $28 \%$ ), en Afrique centrale et de l'Est (28\%), en Afrique de l'Ouest (19\%), en Amérique du Sud (18\%) et en Afrique australe (6\%). La durée médiane de la colonisation après le voyage était de 30 jours (intervalle de confiance $[I C]$ à $95 \%$ : 29 à 33 jours). Toutefois, 11,3\% des sujets étaient toujours colonisés après 12 mois, ce qui souligne l'importance de vérifier les antécédents de voyages d'une personne au cours de l'année précédente. De nombreuses autres études de plus petite envergure ont également évalué le risque de contraction d'une EP-BLSE lors d'un voyage (7). Le risque moyen de colonisation par des EP-BLSE après un voyage est de 643 pour 1000 voyageurs revenant du sous-continent indien, de 340 pour 1000 voyageurs revenant d'Afrique, et de 186 pour 1000 voyageurs revenant d'Amérique centrale et du Sud (Figure 1). Bien que la majorité des études se soient penchées sur le risque de colonisation par des organismes pharmacorésistants, les voyages récents ont été associés à un

Figure 1 : Nombre d'entérobactéries productrices de bêta-lactamases à spectre étendu (EP-BLSE) et d'entérobactéries résistantes aux carbapénèmes (ERC) pour 1000 voyageurs, selon la région visitée

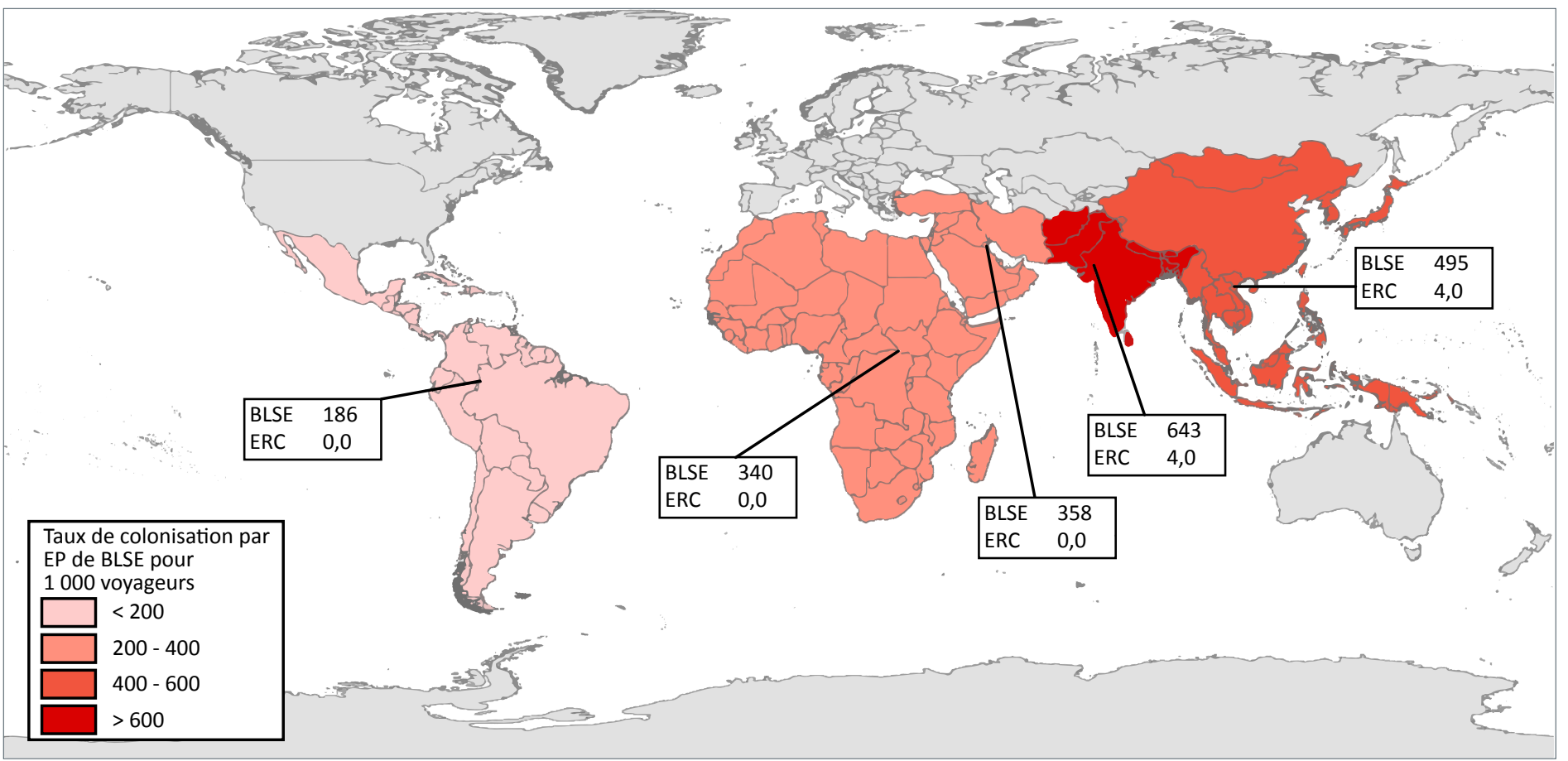

Abréviations : BLSE, bêta-lactamases à spectre étendu; EP, entérobactéries productrices; $E R C$, entérobactéries résistantes aux carbapénèmes; <, inférieur à; >, supérieur à Note : Les données de la figure sont tirées de la moyenne pondérée des études publiées. Figure modifiée avec l'autorisation de Schwartz et Morris (7) 
risque accru d'infections des voies urinaires par des EP-BLSE (811) et de bactériémie causée par des EP-BLSE après une biopsie de la prostate par voie transrectale (12).

\section{Entérobactéries résistantes aux carbapénèmes (ERC)}

Les infections par ERC, qui ont aussi augmenté à travers le monde, soulèvent des préoccupations particulières en raison des options thérapeutiques limitées et des taux élevés de mortalité liée aux infections, lesquels varient de 40 à $70 \%(13,14)$. Les trois principales classes d'entérobactéries productrices de carbapénémases qui confèrent une résistance aux carbapénèmes présentent une épidémiologie régionale distincte (15):

- Klebsiella pneumoniae productrice de carbapénémase (KPC) est l'entérobactérie productrice de carbapénémases la plus courante en Amérique du Nord

- les entérobactéries productrices de carbapénémases de type OXA-48 sont typiques de la Turquie et des régions avoisinantes

- la New Delhi métallo-bêta-lactamase-1 (NDM-1) a initialement été associée aux personnes ayant reçu des soins médicaux sur le sous-continent indien, mais elle est désormais observée sur tous les continents (16)

Le Programme canadien de surveillance des infections nosocomiales (PCSIN) a récemment caractérisé les entérobactéries productrices de carbapénémases signalées entre 2010 et 2014 dans des hôpitaux canadiens. L'incidence était de 0,07 cas pour 1000 admissions; la KPC et la NDM-1 étant les plus courantes. De nombreuses personnes infectées avaient des antécédents de voyages internationaux. L'Inde était la destination la plus fréquente; $31 \%$ des cas ont déclaré avoir visité ce pays au cours des 12 mois précédents (17). Cependant, le risque de contracter une ERC lors d'un voyage est considérablement plus faible que le risque de contracter une EP-BLSE (Figure 1).

\section{Gène $m c r-1$ de résistance à la colistine}

Le gène $m c r-1$ de résistance à la colistine à médiation plasmidique récemment décrit, qui peut être associé à d'autres mécanismes de résistance à gram négatif, suscite des préoccupations supplémentaires. La colistine est l'une des rares options antibiotiques contre les ERC; toutefois, elle présente un risque important de néphrotoxicité et de neurotoxicité. Initialement découvert en Chine dans des isolats cliniques animaux et humains, le gène mcr-1 a depuis été signalé dans des isolats cliniques du monde entier (18). Une récente étude hollandaise a révélé que les échantillons de selles de $5 \%$ des voyageurs de longue distance contenaient le gène $\mathrm{mcr}-1$. Ces voyageurs avaient principalement visité l'Asie du Sud-Est ou l'Afrique australe (19).
Les voyages jouent également un rôle dans la propagation d'autres organismes bactériens pharmacorésistants, comme les espèces de Salmonelle, Shigella et Campylobacter, qui ont été étudiés ailleurs (7).

\section{Quels sont les facteurs de risque associés à l'acquisition d'organismes pharmacorésistants au cours d'un voyage?}

\section{Recours à des soins de santé}

L'accès récent à des soins de santé à l'étranger a été recensé comme un facteur de risque de contraction d'un organisme pharmacorésistant. D'après l'évaluation par le PCSIN des patients canadiens présentant des ERC, $86 \%$ de ceux dont les antécédents de voyages étaient connus avaient obtenu des soins médicaux à l'étranger (17). L'association entre l'accès à des soins de santé lors d'un voyage et la présence d'organismes pharmacorésistants a aussi été observée dans un certain nombre d'études européennes (20-27). Parmi les voyageurs qui rentraient chez eux après avoir été hospitalisés à l'étranger, la colonisation par tout organisme multirésistant variait de 7 \% (21) à $29 \%$ (23). Dans ces études, les organismes multirésistants étaient définis comme étant des EP-BLSE, des ERC, d'autres organismes multirésistants à gram négatif, le staphylocoque doré résistant à la méthicilline et l'entérocoque résistant à la vancomycine. Le risque le plus élevé concernait les patients directement transférés ou rapatriés d'hôpitaux à l'étranger, comparativement à ceux non directement rapatriés (rapport de cotes $[R C]=7,4$; IC à $95 \%: 2,1$ à 25,2) (27). Les autres facteurs de risque comprennent les hospitalisations prolongées à l'étranger $(20,21)$, les antécédents d'intervention chirurgicale à l'étranger (22), les admissions dans une unité à risque élevé (c.-à-d. unité de soins intensifs) (21), les visites dans un pays tropical ou subtropical (particulièrement en Asie du Sud) $(21,24)$ et la prise d'antibiotiques pendant une hospitalisation (21-23).

Même si ces études comprenaient des voyageurs ayant été hospitalisés pour une raison prévue ou imprévue, ce risque pourrait s'appliquer aux touristes médicaux, c'est-à-dire aux voyageurs qui se rendent à l'étranger dans le but précis de recevoir des soins médicaux (28). D'après les estimations à l'échelle mondiale, environ quatre millions de personnes s'adonnent au tourisme médical chaque année (29). Une enquête canadienne révèle que plus de 63000 patients ont reçu des soins médicaux à l'étranger en 2016 (30). La plupart des touristes médicaux canadiens reçoivent des soins aux É-U, suivis par des pays des Amériques et d'Asie à revenu faible ou moyen (31), notamment des régions aux prises avec des taux élevés de pharmacorésistance. Étant donné le risque de contraction d'organismes pharmacorésistants pendant un voyage, 
particulièrement chez les voyageurs qui accèdent à des soins de santé à l'étranger, les personnes qui envisagent le tourisme médical courent un risque important et souvent sous-estimé.

\section{Diarrhée du voyageur}

La diarrhée du voyageur est causée par l'ingestion d'aliments ou de boissons contaminés par des entéropathogènes bactériens (32). Selon la destination du voyage et les facteurs de l'hôte, l'incidence de la diarrhée pendant un voyage peut varier de 10 à $40 \%$. Dans plusieurs études menées auprès de voyageurs ayant contracté des organismes pharmacorésistants à l'étranger, on a constaté que la diarrhée était un facteur de risque important, en particulier en ce qui a trait à l'acquisition d'une EP-BLSE. La diarrhée du voyageur est associée à une augmentation d'environ 2 à 3 fois du risque de contraction d'une EP-BLSE à l'étranger $(6,33,34)$. Dans le cadre d'une étude menée auprès de voyageurs finlandais, le risque d'acquisition d'une EP-BLSE était de $11 \%$ chez ceux qui n'ont pas eu la diarrhée du voyageur, de $21 \%$ chez ceux qui ont eu la diarrhée du voyageur, mais n'ont pas pris d'antibiotiques, et de $37 \%$ chez ceux qui ont eu la diarrhée du voyageur et qui ont été traités par des antibiotiques (33).

\section{Exposition à des antibiotiques}

Les antibiotiques exercent une pression sélective sur les organismes indigènes qui colonisent les intestins, augmentant le risque que des organismes pharmacorésistants contractés à l'étranger soient incorporés au microbiome. On a démontré à plusieurs reprises que l'antibiothérapie présente un risque pour les voyageurs. Dans l'étude hollandaise susmentionnée menée auprès de voyageurs ayant contracté des EP-BLSE à l'étranger, l'utilisation d'antibiotiques a été associée à un risque plus de deux fois supérieur de contracter ces organismes pharmacorésistants ( $R C=2,7$; IC à $95 \%: 1,8$ à 4,0) (6). Dans le cadre d'une étude finlandaise, $21 \%$ des personnes n'ayant reçu aucun traitement, $20 \%$ de celles traitées uniquement par le lopéramide (antidiarrhéique), $40 \%$ de celles traitées uniquement par des antibiotiques, et $71 \%$ de celles traitées par le lopéramide et des antibiotiques ont ensuite été colonisées par une EP-BLSE (35).

Parmi les voyageurs hospitalisés à l'étranger, le risque associé à une antibiothérapie était également prononcé, le risque d'être colonisé par un organisme multirésistant étant 11 fois plus élevé ( $R C=10,7$; IC à $95 \%: 4,2$ à 27,3) que chez les personnes n'ayant pas voyagé à l'étranger. Toutefois, selon cette étude, le fait d'être hospitalisé à l'étranger sans recevoir d'antibiotiques n'était pas un facteur de risque de colonisation par un organisme multirésistant (26). L'importance d'une exposition à des antibiotiques durant une hospitalisation associée à un voyage est corroborée par une vaste étude menée en Finlande, dans laquelle le risque de colonisation par un organisme multirésistant était significativement accru chez les personnes recevant des antibiotiques ( $R C=3,2$; IC à $95 \%$ de 2,3 à 4,5) (23). De même, une étude menée aux Pays-Bas a révélé une multiplication par 2,5 à 3,4 fois du risque de colonisation par un organisme multirésistant à gram positif chez les personnes ayant été traitées par des antibiotiques pendant leur hospitalisation à l'étranger (20).

\section{Que peuvent faire les cliniciens pour réduire autant que possible les risques chez les voyageurs canadiens?}

En comprenant le risque de résistance aux antimicrobiens associé aux voyages, les professionnels de la santé seront plus en mesure d'intégrer des méthodes visant à améliorer la prise en charge et à réduire la transmission d'organismes pharmacorésistants, ainsi que d'informer le public pour qu'il puisse prendre des décisions éclairées (Figure 2). Parmi les occasions qui se présentent aux cliniciens, citons les suivantes:

- avant les voyages, informer les patients du risque de contraction d'un organisme pharmacorésistant en donnant des conseils personnalisés selon l'itinéraire et la destination du patient (Figure 1)

- informer les patients des risques liés à l'accès non prévu à des soins de santé à l'étranger; réduire autant que possible le risque par une immunisation avant le voyage et des conseils sur la façon de prévenir la diarrhée du voyageur et d'éviter les activités à risque élevé

- informer les patients des risques liés au tourisme médical, en donnant des conseils personnalisés selon I'itinéraire et la destination du patient (Figure 1)

- lorsqu'on envisage la prescription d'antibiotiques pour prévenir la diarrhée du voyageur avant le voyage, considérer le risque de contraction d'une EP-BLSE, et comprendre que les récentes lignes directrices encouragent uniquement la dispensation de soins de soutien dans les cas de diarrhée du voyageur légère, et que l'antibioprophylaxie de la diarrhée du voyageur est indiquée uniquement chez certains patients présentant un risque élevé de complications (36)

- tenir compte des voyages récents (dans les 12 mois précédents) lors du choix d'un traitement antimicrobien empirique pour les patients qui ont une infection grave (considérer les patients ayant voyagé en Asie,

Figure 2 : Occasions de prise en charge du risque de contraction d'organismes pharmacorésistants au cours d'un voyage

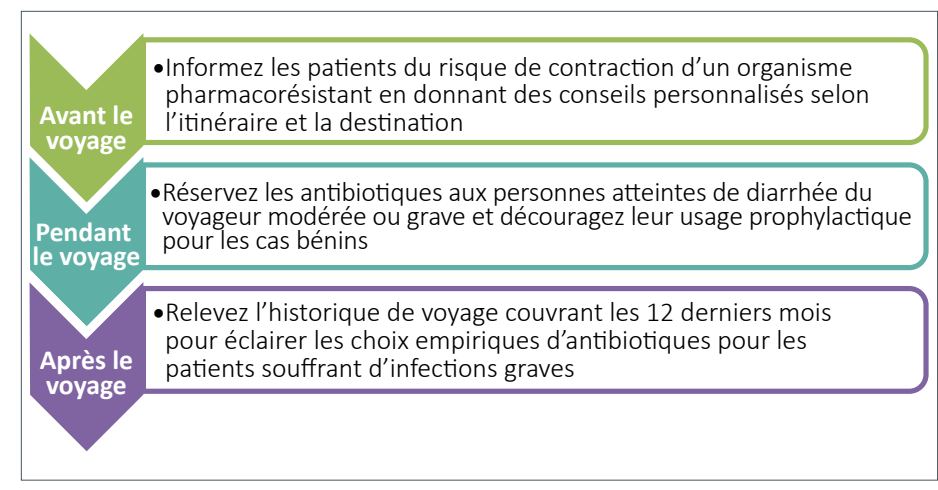


particulièrement sur le sous-continent indien, comme présentant un risque très élevé d'être porteurs d'entérobactéries pharmacorésistantes)

\section{Conclusion}

Les voyages à l'étranger posent un risque important de contraction d'un organisme pharmacorésistant. L'Asie et le sous-continent indien, en particulier, présentent les risques les plus élevés de contracter une EP-BLSE ou une ERC.

Les soins médicaux, la diarrhée du voyageur et l'utilisation d'antibiotiques à l'étranger viennent augmenter les risques pour les voyageurs. Les professionnels de la santé peuvent jouer un rôle important dans la réduction du risque chez les voyageurs, au moyen de conseils, de la prescription d'antibiotiques et de la personnalisation du traitement empirique des patients qui présentent des infections graves et qui ont voyagé récemment.

\section{Conflit d'intérêts}

Aucun.

\section{Remerciements}

Nous souhaitons remercier Sean Marshall, de Santé publique Ontario, pour la création de la carte.

\section{Références}

1. O'Neill J. Review on antimicrobial resistance antimicrobial resistance: tackling a crisis for the health and wealth of nations. London: Review on Antimicrobial Resistance; 2014. https://amr-review.org/sites/default/files/AMR\%20 Review\%20Paper\%20-\%20Tackling\%20a\%20crisis\%20for\%20 the\%20health\%20and\%20wealth\%20of\%20nations_1.pdf

2. ResistanceMap. Washington (DC): Center for Disease Dynamics, Economics \& Policy (CDDEP). https:// resistancemap.coldep.org/

3. Annual Review IA. 2018. Geneva: International Air Transport Association; 2018. https://www.iata.org/media/ annual-report-2018/index.html

4. Schwaber MJ, Navon-Venezia S, Kaye KS, Ben-Ami R, Schwartz D, Carmeli Y. Clinical and economic impact of bacteremia with extended- spectrum-bet a-lactamase-producing Enterobacteriaceae. Antimicrob Agents Chemother 2006 Apr;50(4):1257-62. DOI PubMed

5. Xu L, Sun X, Ma X. Systematic review and meta-analysis of mortality of patients infected with carbapenem-resistant Klebsiella pneumoniae. Ann Clin Microbiol Antimicrob 2017 Mar;16(1):18. DOI PubMed
6. Arcilla MS, van Hattem JM, Haverkate MR, Bootsma MC, van Genderen PJ, Goorhuis A, Grobusch MP, Lashof AMO, Molhoek N, Schultsz C, Stobberingh EE, Verbrugh HA, de Jong MD, Melles DC, Penders J. Import and spread of extended-spectrum ß-lactamase-producing Enterobacteriaceae by international travellers (COMBAT study): a prospective, multicentre cohort study. Lancet Infect Dis 2017 Jan;17(1):78-85. DOl PubMed

7. Schwartz KL, Morris SK. Travel and the spread of drug-resistant bacteria. Curr Infect Dis Rep 2018 Jun;20(9):29. DOI PubMed

8. Osthoff M, McGuinness SL, Wagen AZ, Eisen DP. Urinary tract infections due to extended-spectrum beta-lactamaseproducing Gram-negative bacteria: identification of risk factors and outcome predictors in an Australian tertiary referral hospital. Int J Infect Dis 2015 May;34:79-83. DOI PubMed

9. Søraas A, Sundsfjord A, Sandven I, Brunborg C, Jenum PA. Risk factors for community-acquired urinary tract infections caused by ESBL-producing enterobacteriaceae--a case-control study in a low prevalence country. PLoS One 2013 Jul;8(7):e69581. DOI PubMed

10. Talan DA, Takhar SS, Krishnadasan A, Abrahamian FM, Mower WR, Moran GJ; EMERGEncy ID Net Study Group. Fluoroquinolone-resistant and extended-spectrum beta-lactamase-producing Escherichia coli infections in patients with pyelonephritis, United States(1). Emerg Infect Dis 2016 Sep;22(9): DOI PubMed

11. Tham J, Odenholt I, Walder M, Andersson L, Melander E. Risk factors for infections with extended-spectrum beta-lactamase-producing Escherichia coli in a county of Southern Sweden. Infect Drug Resist 2013 Sep;6:93-7. DOI PubMed

12. Patel U, Dasgupta P, Amoroso P, Challacombe B, Pilcher J, Kirby R. Infection after transrectal ultrasonography-guided prostate biopsy: increased relative risks after recent international travel or antibiotic use. BJU Int 2012 Jun;109(12):1781-5. DOI PubMed

13. Ben-David D, Kordevani R, Keller N, Tal I, Marzel A, Gal-Mor O, Maor Y, Rahav G. Outcome of carbapenem resistant Klebsiella pneumoniae bloodstream infections. Clin Microbiol Infect 2012 Jan;18(1):54-60. DOI PubMed

14. Friedman ND, Carmeli Y, Walton AL, Schwaber MJ. Carbapenem-resistant Enterobacteriaceae: a strategic roadmap for infection control. Infect Control Hosp Epidemiol 2017 May;38(5):580-94. DOI PubMed 
15. van Duin D, Doi Y. The global epidemiology of carbapenemase-producing Enterobacteriaceae. Virulence 2017 May;8(4):460-9. DOI PubMed

16. Wilson ME, Chen LH. NDM-1 and the role of travel in its dissemination. Curr Infect Dis Rep 2012 Jun;14(3):213-26. DOI PubMed

17. Mataseje LF, Abdesselam K, Vachon J, Mitchel R, Bryce E, Roscoe D, Boyd DA, Embree J, Katz K, Kibsey P, Simor AE, Taylor G, Turgeon N, Langley J, Gravel D, Amaratunga K, Mulvey MR. Results from the Canadian Nosocomial Infection Surveillance Program on Carbapenemase-Producing Enterobacteriaceae, 2010 to 2014. Antimicrob Agents Chemother 2016 Oct;60(11):6787-94. DOI PubMed

18. Mediavilla JR, Patrawalla A, Chen L, Chavda KD, Mathema B, Vinnard C, Dever LL, Kreiswirth BN. Colistin- and carbapenem-resistant Escherichia coli harboring mcr1 and blaNDM-5, causing a complicated urinary tract infection in a patient from the United States. MBio 2016 Aug;7(4):e01191-16. DOl PubMed

19. von Wintersdorff CJ, Wolffs PF, van Niekerk JM, Beuken E, van Alphen LB, Stobberingh EE, Oude Lashof AM, Hoebe CJ, Savelkoul PH, Penders J. Detection of the plasmid-mediated colistin-resistance gene mcr-1 in faecal metagenomes of Dutch travellers. J Antimicrob Chemother 2016 Dec;71(12):3416-9. DOI PubMed

20. Kaiser AM, Schultsz C, Kruithof GJ, Debets-Ossenkopp Y, Vandenbroucke-Grauls C. Carriage of resistant microorganisms in repatriates from foreign hospitals to The Netherlands. Clin Microbiol Infect 2004 Nov;10(11):972-9. DOI PubMed

21. Josseaume J, Verner L, Brady WJ, Duchateau FX. Multidrug-resistant bacteria among patients treated in foreign hospitals: management considerations during medical repatriation. J Travel Med 2013 Jan-Feb;20(1):22-8. DOI PubMed

22. Kaspar T, Schweiger A, Droz S, Marschall J. Colonization with resistant microorganisms in patients transferred from abroad: who needs to be screened? Antimicrob Resist Infect Control 2015 Jul;4:31. DOI PubMed

23. Khawaja T, Kirveskari J, Johansson S, Väisänen J, Djupsjöbacka A, Nevalainen A, Kantele A. Patients hospitalized abroad as importers of multiresistant bacteria-a cross-sectional study. Clin Microbiol Infect 2017 Sep;23(9):673.e1-8. DOI PubMed
24. Mutters NT, Günther F, Sander A, Mischnik A, Frank U. Influx of multidrug-resistant organisms by country-to-country transfer of patients. BMC Infect Dis 2015 Oct;15:466. DOI PubMed

25. Nemeth J, Ledergerber B, Preiswerk B, Nobile A, Karrer $S$, Ruef C, Kuster SP. Multidrug-resistant bacteria in travellers hospitalized abroad: prevalence, characteristics, and influence on clinical outcome. J Hosp Infect 2012 Dec;82(4):254-9. DOI PubMed

26. Angue $M$, Allou N, Belmonte $O$, Lefort $Y$, Lugagne N, Vandroux D, Montravers P, Allyn J. Risk factors for colonization with multidrug-resistant bacteria among patients admitted to the intensive care unit after returning from abroad. J Travel Med 2015 Sep-Oct;22(5):300-5. DOI PubMed

27. Birgand G, Armand-Lefevre L, Lepainteur M, Lolom I, Neulier C, Reibel F, Andremont A, Lucet JC. Introduction of highly resistant bacteria into a hospital via patients repatriated or recently hospitalized in a foreign country. Clin Microbiol Infect 2014 Nov;20(11):O887-90. DOI PubMed

28. Lunt N, Carrera P. Medical tourism: assessing the evidence on treatment abroad. Maturitas 2010 May;66(1):27-32. DOI PubMed

29. Smith R, Martínez Álvarez M, Chanda R. Medical tourism: a review of the literature and analysis of a role for bi-lateral trade. Health Policy 2011 Dec;103(2-3):276-82. DOI PubMed

30. Ren F, Labrie Y. Leaving Canada for Medical Care. Fraser Research Bulletin. Vancouver (BC):Fraser Institute; 2017.

31. Runnels V, Labonté R, Packer C, Chaudhry S, Adams O, Blackmer J. Canadian physicians' responses to cross border health care. Global Health 2014 Apr;10:20. DOI PubMed

32. Steffen R, Hill DR, DuPont HL. Traveler's diarrhea: a clinical review. JAMA 2015 Jan;313(1):71-80. DOI PubMed

33. Kantele A, Lääveri T, Mero S, Vilkman K, Pakkanen SH, Ollgren J, Antikainen J, Kirveskari J. Antimicrobials increase travelers' risk of colonization by extended-spectrum betalactamase-producing Enterobacteriaceae. Clin Infect Dis 2015 Mar;60(6):837-46. DOI PubMed

34. Vading $M$, Kabir MH, Kalin M, Iversen A, Wiklund S, Nauclér P, Giske CG. Frequent acquisition of low-virulence strains of ESBL-producing Escherichia coli in travellers. J Antimicrob Chemother 2016 Dec;71(12):3548-55. DOI PubMed 
35. Kantele A, Mero S, Kirveskari J, Lääveri T. Increased risk for ESBL-producing bacteria from co-administration of loperamide and antimicrobial drugs for travelers' diarrhea. Emerg Infect Dis 2016 Jan;22(1):117-20. DOI PubMed
36. DuPont HL, Steffen R. Use of antimicrobial agents for treatment and prevention of travellers' diarrhoea in the face of enhanced risk of transient fecal carriage of multi-drug resistant enterobacteriaceae: setting the stage for consensus recommendations. J Travel Med 2017 Aug;24(Suppl_1):S57-S62. DOI PubMed

Relevé des maladies transmissibles au Canada

\section{LES VOYAGES INTERNATIONAUX AUGMENTENT LE RISQUE} D'EXPOSITION AUX BACTÉRIES PHARMACORÉSISTANTES

\section{QUEL est le risque?}

Les entérobactéries résistantes sont les plus courantes. Elles peuvent entraîner:

- la diarrhée du voyageur

- la bronchite ou la pneumonie

- I'infection des voies urinaires

Les voyageurs peuvent également devenir porteurs de la bactérie et la transmettre à d'autres individus.

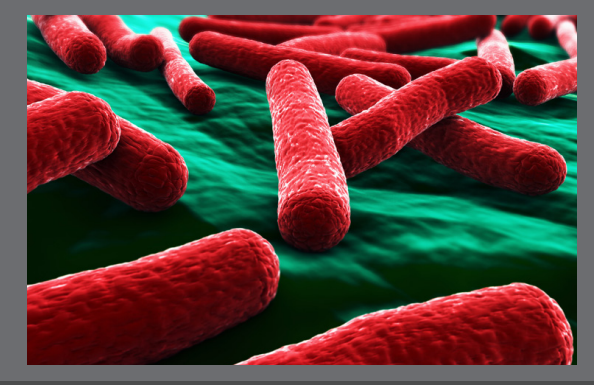

\section{OÙ est le risque?}

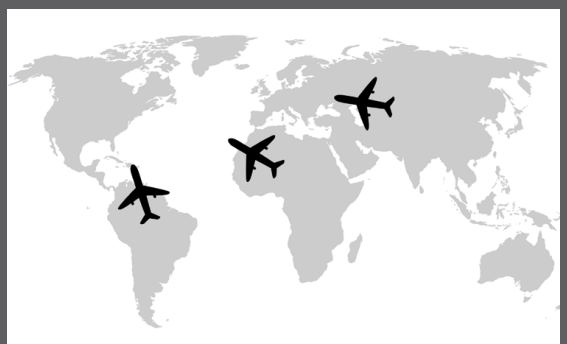

Le risque le plus élevé touche:

- I'Asie, notamment au sud

Mais Aussi:

- I'Afrique et le Moyen Orient

- les Caraïbes et I'Amérique centrale

- I'Amérique du Sud

\section{Pratiques exemplaires}

Établir les antécédents de voyages durant la dernière année

- en particulier chez ceux dont l'infection ne répond pas aux antibiotiques

Informez les patients sur:

- les zones à risque, l'hygiène des mains et les pratiques alimentaires sûres

- le traitement symptomatique de la diarrhée légère

- I'utilisation minimale des services de santé

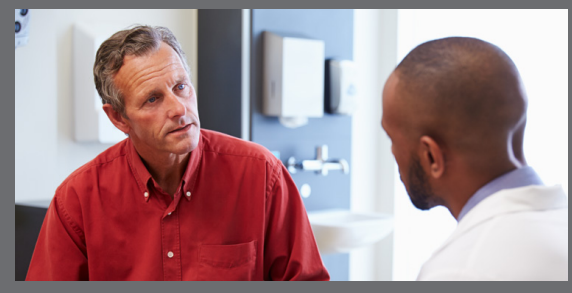

